ANNALES

POLONICI MATHEMATICI

XXI (1969)

\title{
Topology of Laplace transformable functions
}

by 'I. K. MukherJeE (Jadavpur) and S. GaNGuly (Barasat)

Introduction. We consider here the set of all functions that are Laplace transformable with regard to their structure both algebraic and topological. Dutta [2] has revealed some very interesting features in his recent work but, for the sake of convenience, he has considered both the object and the image spaces of transformable function as $L_{2}$-spaces. But Doetsch [1] has pointed out that they are not $L_{2}$-spaces Sneddon [3] remarked that the spaces of transformable functions are Banach spaces. We shall study certain topological properties of the set of Laplace transformable functions with the help of a metric which is natural in a sense to be made clear subsequently.

Let $s_{f}$ denote the abscissa of convergence (see [1]) for a Laplace transformable function $f$, as such for all $s>s_{f}, \int_{0}^{\infty} e^{-x x} f d x$ exists in the Lebesgue sense and is finite, i.e. $e^{-x} f \in L_{1}[0, \infty)=L$. Evidently we cannot assert that $\int_{0}^{\infty} e^{-s_{f x}} f d x$ will exist and will be finite. In fact, $s_{f}$ can be obtained by a Dedekind cut and as such the behaviour at $8_{f}$ cannot be ensured. It is easy to see that there is no loss of generality if we restrict $s_{f}$ in $[0, \infty)$ since a function is already in a $L_{1}$-space if its abscissa of convergence is less than zero. For our convenience we use L.T. for Laplace transformable functions : throughout this paper.

\section{Algebraic Structure.}

THEOREM. The L.T. - set is an Abelian group with respect to the operation of addition (in the usual sense).

Proof. Let $f_{1}$ and $f_{2}$ be two functions and let us suppose that they belong to L.T.-set, i.e. there exist $s_{1}$ and $s_{2}$ such that $\int_{0}^{\infty} e^{-s_{1} x} f_{1} d x$ and $\int_{0}^{\infty} e^{-s_{2} x} f_{2} d x$ exist. Evidently, if we take $s=\max ^{m}\left(s_{1}, s_{2}\right), \int_{0}^{\infty} e^{-s x}\left(f_{1}+f_{2}\right) d x$ exists. Thus the set is closed for addition. The associative property is evident. The null element and the additive inverse are respectively the 
ordinary zero and $-f(x)$. Since $f(x)$ is L.T., $-f(x)$ is also so. The commutative property is obvious. Hence the theorem. system.

It becomes now very easy to verify that our L.T.-set is a linear

Note 1. If we consider only the positive L.T.-set, the set becomes only an Abelian semi-group.

We now define certain symbols which we use throughout. $C_{\mathrm{s}}$ - the class of all functions in the L.T.- set such that $s_{f}=s$. Then $C_{s}=C_{s}^{1}, C_{s}^{2}$, where

$$
\left\{f: e^{-s x} f \in L_{1}[0, \infty)\right\}==C_{s}^{1} \quad \text { and } \quad\left\{f: e^{-s x} f \in L_{1}[0, \infty)\right\}=C_{s}^{2} .
$$

If $s=0$, then $C_{0}=C_{0-} \cup C_{0+}$, where $C_{0-}=\left\{f: s_{f}<0\right\}$ and $C_{0+}$ $=\left\{f: s_{f}=0\right\}=C_{0}^{1} \cup C_{0}^{2}$. Hence $C_{0}=C_{0-} \cup C_{0}^{1} \cup C_{0}^{2}$. Thus the L.T. - set $=\bigcup C_{r}=\left(\bigcup_{r} C_{r}^{1}\right) \cup\left(C_{r}^{2}\right)=C^{1} \cup C^{2}(r$ being real and $\geqslant 0)$.

Again evidently for $s>0$ and $t>0, C_{t}=e^{(t-s) x} C_{s} ;$ for $s=0$, $C_{t}=e^{l x} C_{0+}$, i.e. $C_{l}^{1}=e^{t x} C_{0+}^{1}$ and $C_{l}^{2}=-e^{t x} C_{0+}^{2}$. We define $L^{s}[0, \infty)$ as a Banach space with the norm

$$
\|f\|=\int_{0}^{\infty} e^{-8 x}|f(x)| d x<\infty .
$$

The norm introduces the metric

$$
\varrho(f, g)=\int_{0}^{\infty} e^{-s x}|f(x) \cdots g(x)| d x<\infty .
$$

DEFINITION of METRIC. Let $f$ and $g$ be two functions belonging to the L.T.-set. We define the metric as follows:

$$
\varrho(f, g)==\left|s_{f}-s_{g}\right|+\frac{\int_{0}^{\infty}\left|e^{-s \rho x} f-e^{-g_{g} x} g\right| d x}{1+\int_{0}^{\infty}\left|e^{-s f x} f-e^{-s_{g} x} g\right| d x} .
$$

Note 2. $s_{f}$ can be looked upon as a functional on the I.T. - set. It may be seen that in this topology $s_{1}$ is a continuous functional. ditions:

We may now show that the metric satisfies all the required con-

(i) If $f=:$, evidently $\varrho(f, g)=0$; conversely, if $\varrho(f, g) \cdots 0$,

$$
\left|s_{f}-s_{g}\right|+\frac{\int_{0}^{\infty}\left|e^{-s_{f} x} f-e^{-s_{g} x} g\right| d x}{1+\int_{0}^{\infty}\left|e^{-s_{f} x} f-e^{-s_{g} x} g\right| d x}
$$


The two portions being separately positive, they must vanish separately, i.e. $\left|s_{f}-s_{g}\right|==0$ giving

$$
s_{f}=s_{g} \quad \text { and } \quad \int_{0}^{\infty}\left|e^{-s_{f} x} f-e^{-s_{g} x} g\right| d x=0,
$$

1.e. $e^{-s_{f} x} f=e^{-s_{y} x} y$.

But $s_{f}=s_{g} ;$ hence $f=g$.

The property of symmetry and transitivity being very obvious it follows that $\varrho$ is a metric.

Note $3 . s_{f_{n}} \rightarrow s_{f}$ if

$$
\varrho\left(f_{n}, f\right) \rightarrow 0 \quad \text { and } \quad\left\{\begin{array}{l}
f_{n} \rightarrow f \\
s_{f_{n}} \rightarrow s_{f}
\end{array}\right\} \Leftrightarrow g_{n} \rightarrow f
$$

where $g_{n} \in C_{s_{f}}, g_{n}$ being equal to $\exp \left\{\left(s_{f_{n}}-s_{f}\right) x\right\} f_{n}(x)$.

This shows that if $f_{n} \rightarrow f$, then there is a sequence $g_{n} \in C_{B_{f}}$ such that $g_{n} \rightarrow f$ so that any convergent sequence can always be taken to be confined in a given class $C_{s}$.

Note 4. $s_{f}$ being a continuous linear functional on the L.T.-space and $C_{s}$ being equal to $\left\{f: s_{f}=s\right\}$ it follows that every $C_{s}$ is closed.

Note 5 . In $C_{s}^{1}$ the metric becomes $\varrho(f, g)=\int_{0}^{\infty} e^{-s x}|f(x)-g(x)| d x$ so that the relative topology in $C_{y}^{1}$ induced by the L.T.-space is the same as the one induced by $L_{s}[0, \infty)$. Suppose now that $C_{s}$ is given with its topology as induced by $L_{s}[0, \infty)$; then one way of metrising $\cup C_{s}^{1}$ so that each subspace $C_{s}^{1}$ has the same relative topology as above, is given by our metric.

Note 6. The usual uniform convergence in L.T.-space does not imply convergence as induced by the above metric.

We now study $C_{0}$ alone, but these considerations can easily be extended to $C_{s}$.

In $C_{0}$ we define a relation between $f$ and $g$ :

$$
f R g \text { iff } \int_{0}^{\infty}|f(x)-g(x)| d x<\infty .
$$

Evidently $h$ is an equivalence relation. Obviously since $g(x)=f(x)+$ $+[g(x)-f(x)]$, it follows that $|g(x)| \leqslant|f(x)-g(x)|+|f(x)|$, where $(g(x)-$ $-f(x)) \epsilon L_{1}$. It follows that $C_{0}$ is partitioned into disjoint classes and each class is of the form $\left(f+L_{1}\right)$, where $\int_{0}^{\infty}|f(x)| d x=\infty$. The distance between any two elements of two classes is 1 . In fact, these are elements of the factor space of $C_{0}$ with respect to $L_{1}$ in $C_{0}$. Thus the factor space in its 
quotient topology is discrete. This is not very unnatural since our metric has not made any use of the crucial property of a function $f \in C_{0}^{2}$, i.e. $\int_{0}^{\infty} e^{-\varepsilon x}|f(x)| d x<\infty$ for every $\varepsilon>0$.

It appears that our metric is not sensitive enough for studying the I.'T. - set. Perhaps we can consider a different metric in this way:

$$
\varrho(f, g)=\left|s_{f} \cdots s_{g}\right|+\sum_{n=1}^{a} \frac{1}{2^{n}} \frac{\left\{\int_{0}^{n}|f-g|^{p} d x\right\}^{1 / p}}{\left.1+\left|\int_{0}^{n}\right| f-\left.g\right|^{p} d x\right\}^{1 / p}} .
$$

The metric introduces the $L_{p}$-convergence on each compact subset of reals. Actually, by this metric the distance between two classes does not become unity and the factor space topology will not be discrete.

THEorem. The L.T.-space is complete with our metric.

I set $\left\{f_{n}\right\}$ be a Cauchy sequence in the I.T.-space.

Let $N_{1} \subseteq N, N$ being the set of positive integers, be defined as $N_{1}=\left\{n: g_{n}(x) \in C_{0}^{1}\right\}$ and $N_{2} \subseteq N=\left\{n: g_{n}(x) \in C_{0}^{2}\right\}$.

Then neither $N_{1}$ nor $N_{2}$ can be infinite for that would contradict the fact that $\left\{f_{n}\right\}$ is a Cauchy sequence.

Now

$$
\varrho\left(f_{n}, f_{n+m}\right)=\left|s_{f_{n}}-8_{/_{n+m}}\right|+\frac{\int_{0}^{\infty}\left|\exp \left\{-8_{f_{n}} x\right\} f_{n}-\exp \left\{-s_{f_{n+m}} x\right\} f_{n+m}\right| d x}{1+\int_{0}^{\infty}\left|\exp \left\{-s_{f_{n}} x\right\} f_{n}-\exp \left\{-s_{f_{n+m}} x\right\} f_{n+m}\right| d x} .
$$

But since the real number space is complete and $C_{0} \cup C_{0}^{1}=L_{1}$ is complete, then $g_{n} \rightarrow g$, i.e. $f_{n}(x) \rightarrow\left\{e^{s x} g(x)\right\}$. If $g_{n}, n \geqslant N_{0}$, belongs to $C_{0}^{2}$, then $g_{n} \rightarrow(f)$ for $n \geqslant N_{0}$, where $f \in C_{0}^{2}$. Then $g_{n} \rightarrow f+L_{1}$, i.e.

$$
\int_{0}^{a}\left|\left(g_{n}-f\right)-\left(g_{n+m}-f\right)\right| d x=\int_{0}^{a}\left|g_{n}^{\prime}-g_{n+m}^{\prime}\right| d x \rightarrow 0 .
$$

$L_{1}$ being complete, $g_{n}^{\prime} \rightarrow g^{\prime}, g^{\prime} \in L_{1}$ and $g_{n} \rightarrow f+g^{\prime}$, i.e. $f_{n} \rightarrow e^{\star x}\left(f+g^{\prime}\right) \epsilon$ $\epsilon$ I.T.T. - space.

Hence the L.T. -space is complete.

THEOREM. The L.T.-space is disconnected.

Proof. We know that the L.T.-space $=C^{1} \cup C^{2}$. We have just shown that $C^{1}$ is complete, and so evidently it is closed. Similarly $C^{2}$ is closed.

Hence the L.T.-space is disconnected.

Note 7. Every $C_{8}$ thus becomes disconnected in its relative topology and $C_{s}=C_{s}^{1} \cup C_{s}^{2}$, where $C_{s}^{1}$ and $C_{s}^{2}$ are relatively closed in $C_{s}$. 
Separablutty. Theorem $-C^{1}$ is separable in the relative topology.

Proof. In fact, let $\left\{s_{n}\right\}$ be a countable dense subset of $[0, \infty)$. Then let $\left\{f_{n}\right\}$ be a dense subset of $\left[C_{0-} \cup C_{0+}^{1}\right]$ which in its relative topology is identical with $L_{1}[0, \infty)$. Then $\left\{e^{s_{n} x} f_{m}(x)\right\}$ is a dense subset in $C^{1}$ as can easily be seen.

Hence the proof.

We shall now consider only the positive functions. The continuity of the additive operation can be proved in the following manner:

Let $s_{f}, s_{g}, s_{f_{n}}, s_{g_{n}}$ be the abscissas of convergence for $f, g, f_{n}$ and $g_{n}$ respectively.

Case I. Let $s_{0}>s_{f}$; then there is a neighbourhood of $s_{0}$ in which there is no element of the sequence $s_{f_{n}}$; and, since $s_{g}$ is the limit of $s_{\sigma_{n}}$, this neighbourhood contains all $s_{g_{n}}$ for $n \geqslant n_{0}$.

Hence for all $n \geqslant n_{0}$ the abscissa of convergence of $f_{n}+g_{n}$ is $s_{g_{n}}$ :

$$
\begin{aligned}
\varrho\left(f_{n}+g_{n}, f+g\right)= & \left|s_{g_{n}}-s_{g}\right|+ \\
& +\int_{0}^{\infty}\left|\exp \left\{-s_{g_{n}} x\right\}\left(f_{n}+g_{n}\right)-\exp \left\{-s_{g} x\right\}(f+g)\right| d x \\
\leqslant & \left|s_{g_{n}}-s_{g}\right|+\int_{0}^{\infty}\left|\exp \left\{-s_{g_{n}} x\right\} g_{n}-\exp \left\{-s_{g} x\right\} g\right| d x+ \\
& +\int_{0}^{\infty}\left|\exp \left\{-s_{g_{n}} x\right\} f_{n}-\exp \left\{-s_{g} x\right\} f\right| d x \\
\leqslant & \varrho\left(g_{n}, g\right)+\int_{0}^{\infty} \mid \exp \left\{-s_{y_{n}} x+s_{f_{n}} x\right\} \exp \left\{-s_{f_{n}} x\right\} f_{n}-\cdots \\
& -\exp \left\{-s_{g_{n}} x+s_{f_{n}} x\right\} \exp \left\{-s_{f} x\right\} f \mid d x- \\
& +\int_{0}^{\infty} \mid \exp \left\{-s_{g_{n}} x+s_{f_{n}} x\right\} \exp \left\{-s_{f} x\right\} f- \\
& -\exp \left\{-s_{g} x+s_{f} x\right\} \exp \left\{-s_{f} x\right\} f \mid d x \\
= & \varrho\left(g_{n}, g\right)+\int_{0}^{\infty}\left|\exp \left\{-s_{g_{n}} x+s_{f_{n}} x\right\}\right| \times \\
& \times\left|\exp \left\{-s_{f_{n}} x\right\} f_{n}-\exp \left\{-s_{f} x\right\} f\right| d x+ \\
& +\int_{0}^{\infty}\left|\exp \left\{-s_{f} x\right\} f\right|\left|\exp \left\{-s_{g_{n}} x+s_{f_{n}} x\right\}-\exp \left\{-s_{g} x+s_{f} x\right\}\right| d x \\
\leqslant & \varrho\left(g_{n}, g\right)+\varrho\left(f_{n}, f\right)+\int_{0}^{\infty}\left|\exp \left\{-s_{f} x\right\} f\right| \mid \exp \left\{-s_{g_{n}} x+s_{f_{n}} x\right\} \\
& -\exp \left\{-s_{g} x+s_{f} x\right\} \mid d x \rightarrow 0
\end{aligned}
$$

as $n \rightarrow a$, i.e. $\left(f_{n}+g_{n}\right) \rightarrow(f+g)$ as $n \rightarrow a$. 
Case II $s_{f}=s_{g}$. In this case in every neighbourhood of $s_{f}$ and similarly in every neighbourhood of $s_{g}$ there are elements of $s_{f_{n}}$ also. But in a certain case $s_{f_{n}+g_{n}}=s_{f_{n}}$, and in other eases $s_{v_{n}}$. But in all cases we have

$$
\begin{aligned}
Q\left(f_{n}: g_{n}, f_{\sqsupset} g\right) & \\
& =\left|s_{f_{n}}--s_{f}\right| \dashv-\int_{0} \exp \left\{-s_{f_{n}} x\right\}\left(f_{n}+g_{n}\right) \cdots \exp \left\{-s_{f} x\right\}(f+g) d x
\end{aligned}
$$

or

$$
\begin{aligned}
\left|s_{a_{n}}-s_{g}\right| & +\int_{0}^{\infty}\left|\exp \left\{-s_{g_{n}} x\right\}\left(f_{n}+g_{n}\right)-\exp \left\{-s_{g} x\right\}(f+g)\right| d x \\
\therefore & \varrho\left(g_{n}, g\right)+\varrho\left(f_{n}, f\right)+ \\
& \quad\left\{\int_{0}^{\infty}\left|\exp \left\{-s_{g} x\right\} g\right|\left|\exp \left\{s_{f_{n}} x+s_{g_{n}} x\right\}-\exp \left\{-s_{f} x+s_{g} x\right\}\right| d x \mid \rightarrow 0\right. \\
& \left\{\left.\int_{0}^{\infty}\left|\exp \left\{-s_{f} x\right\} f\right|\left|\exp \left\{-s_{\sigma_{n}} x+s_{f_{n}} x\right\}-\exp \left\{-s_{g} x+s_{f} x\right\}\right| d x\right|^{\infty}\right.
\end{aligned}
$$

as $n \rightarrow \infty$, i.e. $\left(f_{n}+g_{n}\right) \rightarrow(f+g)$ as $n \rightarrow \infty$.

This shows that $\left(f_{n}+g_{n}\right) \rightarrow(f+g)$ for all $f_{n} \rightarrow f$ and $g_{n} \rightarrow g$. Now the $I_{1}$.'T. - space can be shown not to be a linear metric space with the metric as introduced above. The property that $\alpha_{n} f \rightarrow a f$ is not valid for all funnctions $f$ in the space. In fact, if we consider only the set of all positive functions, then that set will form a topological semi-group.

We express our thanks and gratitude to Prof. Dr. M. Dutta for his kind help and assistance in the preparation of the paper.

\section{References}

[1] G. Doetsch, Theorie und Anwendung der Laplace transformation, 1943.

[2] M. Dutta, On abstract structure of theories of integral transforms, Proc. Nat. Inst. Sci. India 32, A (4) (1966).

[3] I. N. Sneddon, Fourier Transforms, 1951. 